\title{
Adequacy of information transferred at resident sign- out (inhospital handover of care): a prospective survey
}

\author{
S M Borowitz, ${ }^{1}$ L A Waggoner-Fountain, ${ }^{1}$ E J Bass, ${ }^{2}$ R M Sledd ${ }^{2}$
}

\section{See editorial on page 4 \\ ${ }^{1}$ Department of Pediatrics, University of Virginia, \\ Charlottesville, Virginia, USA; \\ ${ }^{2}$ Department of Systems and Information Engineering, \\ University of Virginia, \\ Charlottesville, Virginia, USA}

Correspondence to: Dr S M Borowitz, Division of Pediatric Gastroenterology and Nutrition, University of Virginia Charlottesville, Virginia 22908, USA; Witz@virginia.edu

Accepted 20 May 2007

\begin{abstract}
Background: During sign-out (handover of care), information and responsibility about patients is transferred from one set of caregivers to another. Few residency training programmes formally teach resident physicians how to sign out or assess their ability to sign out, and little research has examined the sign-out process.

Objective: To characterise the effectiveness of the signout process between resident physicians on an acute care ward.
\end{abstract}

Design/methods: Resident physicians rotating on a paediatric acute care ward participated in a prospective study. Immediately after an on-call night, they completed a confidential survey characterising their night on call, the adequacy of the sign-out they received, and where they went to get information they had not received during signout.

Results: 158 of 196 (81\%) potential surveys were collected. On 49/158 surveys (31\%), residents indicated something happened while on call they were not adequately prepared for. In 40/49 instances residents did not receive information during sign-out that would have been helpful, and in 33/40 the situation could have been anticipated and discussed during sign-out. The quality of sign-out lassessed using a five-point Likert scale from $1=$ inadequate to answer call questions to $5=$ adequate to answer call questions) on the nights when something happened the resident was not adequately prepared for were significantly different than the nights they felt adequately prepared (mean (SD) score 3.58 (0.92) and $4.48(0.70) ; p=0.001)$. There were no significant differences in: how busy the nights were; numbers of patients on service at the beginning of the call shift; numbers of admissions during a call shift; numbers of transfers to an intensive care unit; whether residents were "cross-covering" or were members of the general ward team; or whether the resident had cared for the patient previously.

Conclusion: Although sign-out between resident physicians is a frequent activity, there are many times when important information is not transmitted. Analysis of these "missed opportunities" can be used to help develop an educational programme for resident physicians on how to sign out more effectively.

Sign-out (handover of care) is a mechanism of transferring information, responsibility, and authority from one set of caregivers to another set of caregivers. ${ }^{1-4}$ In teaching hospitals, sign-out between resident physicians has a long tradition. In July 2003, duty hour restrictions were instituted for all residency programmes in the USA. Because of the need to reduce the number of hours resident physicians spend in the hospital, the number of sign-outs between resident physicians has increased whereas continuity of care during hospital stay has decreased. ${ }^{5}$ As a result of these changes resident physicians have become increasingly dependent on the exchange of information during sign-out. ${ }^{167}$

Despite the critical importance of sign-out between resident physicians, little research has examined the content, process and effectiveness of resident sign-out. ${ }^{13}$ This study characterises residents' perceptions of the completeness and effectiveness of sign-out on an acute care ward in a children's hospital.

\section{METHODS}

\section{Scope and study population}

The institutional review board of the University of Virginia approved this study and all participants gave informed consent.

We conducted the study on two contiguous general paediatric wards at the University of Virginia Children's Hospital. The general paediatrics service consists of three first year paediatric and/or family medicine residents, two third year paediatric residents, and a paediatric attending physician. Each patient is assigned to a first year and third year resident. The first year resident serves as the child's primary care provider and the third year resident as a supervisor. The entire team undertakes a round every morning. Night coverage is shared by eight residents - the three first year residents and two third year residents on the general paediatric ward rotation and two second year paediatric residents and one first year paediatric resident on other rotations, who "cross-cover" at night. Residents are on call every fourth night. Each night, one first year resident and one second or third year resident are on call and care for all of the patients on the two units during the night shift. The organisational structure of the ward team is depicted in fig 1.

Sign-out for these wards takes place in the Pediatric Library, which is located on one of the units. On weekdays, at 7:00 hours, residents who had been caring for all of the patients on the wards during the night shift meet with the other members of the team to review what happened overnight and hand over care back to the primary team. At 16:00 hours, team members meet the two residents who will be on-call that night and will care for the patients overnight. This is the longest and most comprehensive, and perhaps the most important sign-out session because members of the 
Figure 1 General paediatrics ward team. Non-ward team members who cover the general wards on call. Haem, haematology; onc, oncology.

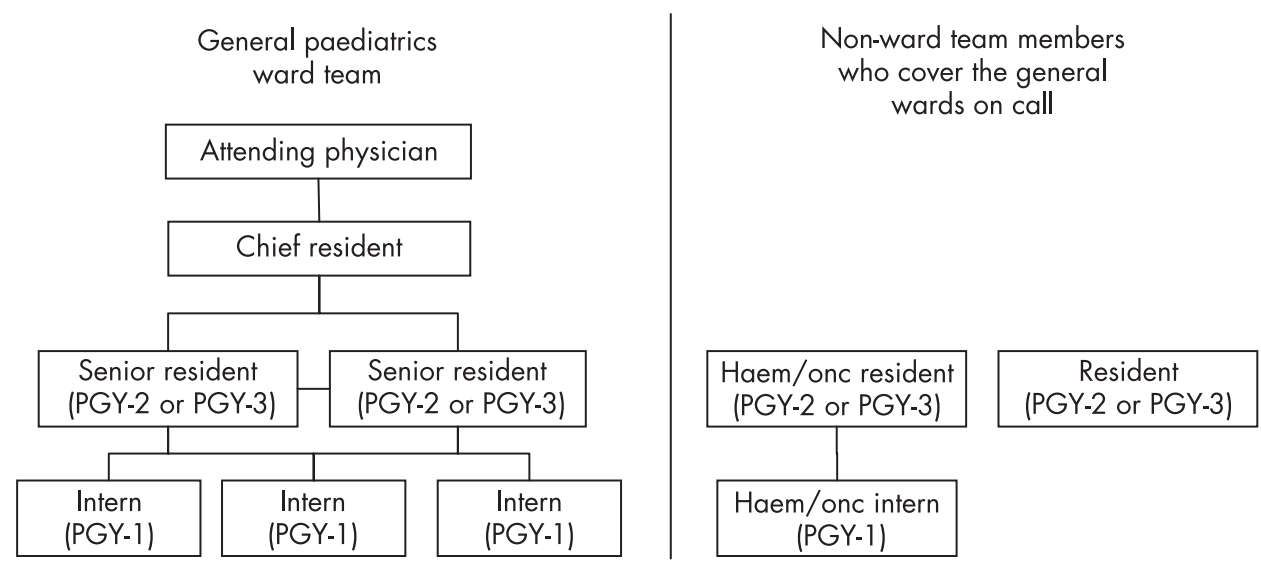

general ward team are handing over care of all the patients on the wards to two physicians who may or may not be members of the primary team. On weekends there is a single sign-out at noon each day, when the pair of residents who had cared for the patients over the previous $24 \mathrm{~h}$ and are ending their shift hand over care to a pair of residents who will assume care of the patients for the following $24 \mathrm{~h}$. For this study, we focused on the 16:00 hours weekday sign-out and weekend sign-outs because they often involve physicians unfamiliar with the patients and are typically the most comprehensive sign-outs. The residents identify the 16:00 hours sign-out as the most important daily sign-out session. For this study the mean (SD) duration of these sign-out sessions was 34.3 (15.5) min (range 11-70 $\mathrm{min})$.

\section{Post-call survey}

After each night that the residents were on call on the two paediatric acute care wards, they completed a confidential printed two-page survey (appendix A). The survey characterised their night on call, the adequacy of the sign-out they received, and where they went to get information they had not received during sign-out. The survey was created by the authors and was based on a conceptual model of handovers of care developed through an institutional quality improvement project and preliminary data obtained by a systems engineer who attended and recorded a number of sign-out sessions. Concurrent with the audio-taped sign-outs, the engineer followed first year physicians through their call period to ascertain what types of questions the residents were asked while they were on call and how they tried to answer those questions.

\section{Data analysis}

We compared continuous variables using unpaired two tailed t tests. Dichotomous variables were compared using the Fisher exact test. Differences were considered statistically different if $p<0.05$. Unless otherwise stated, all results are presented as mean (SD).

\section{RESULTS}

Over 98 days spanning a 4-month period during the winter of 2005-6, each post-call resident received a text page reminding them to complete a survey. During this 4-month period, 26 residents rotated on the wards. All of them completed at least one post-call survey. Of a total potential 196 surveys, 158 (81\%) were completed- $60 \%$ by members of the general paediatric ward team and $40 \%$ by residents who were "cross-covering" on the wards at night or during a weekend. This is very similar to the percentage of night calls covered by residents on the general ward team $(62 \%)$ and residents who were cross-covering (38\%). There were repeated measures both on individual nights of call and by individual residents on different call nights.

In 49/158 (31\%) surveys, resident physicians indicated something happened while they were on call for which they were not adequately prepared. In 40 of these 49 (82\%) instances, they indicated there was information they did not receive during sign-out that would have been helpful to them in caring for a patient overnight, and in 33 of these 40 (82.5\%) instances, they indicated the situation should have been anticipated and discussed during sign-out.

The residents assessed the quality of the sign-out they received using a five-point Likert scale $(1=$ inadequate to answer call questions to $5=$ adequate to answer call questions). Sign-out for nights when something happened for which the residents were not adequately prepared for were rated significantly lower than nights when nothing unexpected happened (3.58 (0.92) and $4.48(0.70)$, respectively; $p=0.001)$. There did not seem to be mitigating factors beyond the quality of the sign-out: there were no differences in how busy the nights were as assessed with a five-point Likert scale ( $1=$ slow to $5=$ busy) (2.93 (1.07) vs 3.25 (1.06); $p=0.08)$, the number of patients on service at the beginning of the call shift (14.85 (4.33) vs 14.33 (4.56); $p=0.49)$, the number of patients admitted during the call shift (4.86 (2.86) vs 4.86 (3.21); $p=0.99)$, or the number of patients they transferred to an intensive care unit during their call shift $(p=0.25)$.

Residents were no more likely to report events they were unprepared for when they were "cross-covering" at night than when they were members of the general paediatric ward team (34.9\% vs $29.0 \%$; $p=0.60$ ). Similarly, when resident physicians reported an event for which they were not prepared, they were just as likely to have cared for that child previously as not $(50 \%$ vs $50 \%$; $p=0.99)$.

On the 40 surveys in which residents indicated there was information they did not receive during sign-out that would have been helpful to them in caring for a patient overnight, they indicated where they went to get information that they had not received during sign-out. Fourteen of the 40 (35\%) residents went to the daily progress note in the chart, however only $3 / 14$ times did they find useful information in the progress note. Smaller numbers went to the chart and reviewed attending physician, consultant or nursing notes, and with each of these notes, they found useful information fewer than half the times. Nine of the $40(22.5 \%)$ residents phoned an attending physician, 
Table 1 Where residents went to get additional information when they did not get necessary information during sign-out $(n=40)$

\begin{tabular}{lcll}
\hline Information sources & Yes (\%) & No (\%) & $\begin{array}{l}\text { Got useful } \\
\text { information }\end{array}$ \\
\hline Went to the chart & $27(67.5)$ & $13(32.5)$ & \\
$\quad$ Went to progress note & $14(35)$ & $26(65)$ & $3 / 14$ \\
Went to attending note & $8(20)$ & $32(80)$ & $3 / 8$ \\
Went to consultant note & $4(10)$ & $36(90)$ & $2 / 4$ \\
Went to nursing notes & $10(25)$ & $30(75)$ & $5 / 10$ \\
$\quad$ Went to other part of the chart & $6(15)$ & $34(85)$ & $4 / 6$ \\
Phoned someone & $17(42.5)$ & $13(57.5)$ & \\
Phoned attending & $9(22.5)$ & $31(77.5)$ & $5 / 9$ \\
Phoned fellow & $10(25)$ & $30(75)$ & $6 / 10$ \\
Phoned chief resident & 0 & $40(100)$ & NA \\
Phoned consultant & $5(12.5)$ & $35(87.5)$ & $3 / 5$ \\
Phoned somebody else & $3(7.5)$ & $37(92.5)$ & $3 / 3$ \\
Went to the computer & $10(25)$ & $30(75)$ & \\
From MIS* & $9(22.5)$ & $31(77.5)$ & $2 / 9$ \\
From CareCast $\dagger$ & $3(7.5)$ & $37(92.5)$ & $1 / 3$ \\
$\quad$ Used clinical judgement & $13(32.5)$ & $27(67.5)$ & NA \\
Asked patient/family & $8(20)$ & $32(80)$ & $3 / 8$ \\
Could not resolve issue & $1(2.5)$ & $39(97.5)$ & NA \\
\hline
\end{tabular}

NA, not applicable.

People could select multiple choices under each subheading.

${ }^{*}$ MIS, Medical Information System (a hospital-wide computerised physician order entry system).

$\dagger$ CareCast, computerised results and document repository.

$10(25 \%)$ phoned a fellow, and $5(12.5 \%)$ phoned a consultant. Approximately half of the time, they obtained useful information from their phone call. In 13 (32.5\%) of 40 instances, residents could not find a clear answer to their question and they used their best clinical judgment to resolve the issue. These results are summarised in table 1 .

\section{DISCUSSION}

The primary objective of any patient sign-out is the accurate transfer of information about the patient's current state and their plan of care. ${ }^{8}$ This transfer of information is crucial for patient safety and successful care. ${ }^{9}$ There are substantial risks of failing to be told, forgetting or misunderstanding information that has been communicated during any patient care handover. $^{71011}$ Incomplete information transfer and incomplete and/ or unclear communication of the plan of care to covering physicians can adversely affect the quality of care. ${ }^{9-12}$ Communication problems are judged to be the most common cause of preventable inhospital disability or death, ${ }^{13}$ and more than $60 \%$ of root causes of sentinel events reported to the Joint Council on Accreditation of Healthcare Organizations (JCAHO) are judged to be due to failures of communication between healthcare personnel. ${ }^{14}$ Resident physicians believe communication difficulties have a major role in the vast majority of medical mishaps they experience. ${ }^{15}$

Our current study further shows that resident sign-out is a point of vulnerability. On nearly one-third of the nights they were on call, resident physicians indicated something happened that they were not adequately prepared for, and in most of these cases, they believed the situation could have been anticipated and should have been discussed during sign-out. Surprisingly, resident physicians were no more likely to report an event they were unprepared for if they were "cross-covering" than if they were a member of the primary team. Similarly, residents were as likely to report an event they were unprepared for if they had cared for the child previously or not. When we reviewed answers to the open-ended question on our survey, three themes emerged about the deficiencies of sign out:

- Sign-out was not useful if the data provided during sign-out were not up to date.

- It is important to include a rationale for the plan of care so that if changes need to be made during a call shift, there is a clear context for how to best make those changes.

- Residents should anticipate problems that may occur during a call shift and provide contingency plans for those potential problems.

Our study has several limitations. While the study was performed prospectively, our principal means of data collection was through post-call surveys during which we asked resident physicians if problems could and should have been anticipated and discussed during sign-out. These types of assessments can introduce considerable hindsight bias. Another potential limitation of our study is that it was performed at a single institution on a single ward service. This may limit the generalisability of our results as there may be unique aspects to this particular acute general paediatric ward service, in that it is geographically located on a single floor and by its very nature, it looks after children who often cannot talk and so there may be an increased importance of caregivers and larger variation in medication doses. Conversely, there are some aspects of the study that may make our results applicable to a wide variety of settings. There is a relatively rapid turnover of patients typical of many paediatric and medical acute ward services in university hospitals. Moreover, the study included paediatric and family medicine residents at various levels of training,

Although we focused on the sign-out process between resident physicians in a teaching hospital, sign-out does not solely take place between physicians in teaching hospitals. Rather, sign-out between physicians takes place in many healthcare settings and continues to take place after residency has been completed. Most community physicians share calls with other physicians. The transfer of care from an on-call physician who has admitted a patient to the hospital during a night or weekend to another physician is a frequent occurrence, especially in large group practices. ${ }^{6}$ Despite the increasing frequency and importance of sign-outs in medical practice, in most settings sign-out remains an informal unstructured process, with great variation and very little standardisation in the type and extent of information exchanged between care providers $^{12} 16$ and in the way and the order with which the information is conveyed. ${ }^{17}$ Even in the same "microenvironment", handovers of care can vary tremendously. ${ }^{18}$ This variability increases the potential for omissions of information and miscommunication ${ }^{17} 19$ and may make it difficult to anticipate what information will be received in handover leading to wasted effort finding the information in other places even if it is covered. It may also make handovers less efficient since the "rules" of the handover have to be negotiated for each handover.

The results of this and other studies indicate sign-out between resident physicians is often inadequate and incomplete. Although no studies have examined the sign-out process between faculty physicians or between physicians in practice, these sign-outs probably have similar shortcomings. This should not be surprising as few training programmes formally teach resident physicians how to sign out and even fewer programmes assess the ability of resident physicians to sign out to their colleagues. ${ }^{414} 19$ Presently, sign-out is learned informally "on the job". Interns and junior residents learn how to sign out by observing more senior residents give sign-out. ${ }^{1419}$ Several 
authors have suggested that residents should be trained to communicate effectively at the time of handovers of care, ${ }^{15} 819$ but there is little evidence to guide the development of such educational programmes and even less evidence on the effectiveness of any training interventions. ${ }^{14}$ While several authors have emphasised different components and strategies to improve sign-out, ${ }^{15819}$ the goals and characteristics of concise and complete sign-out must be defined before any specific curriculum can be created.

Some authors have proposed computer-based sign-out systems as a means of improving the efficiency and quality of resident sign-out, ${ }^{1}{ }^{20} 21$ In the few cases where computer-based sign-out systems have been characterised and evaluated, these systems have been developed as a means of automating existing sign-out processes to make them more efficient for the providers involved. ${ }^{20}{ }^{21}$ Implementation of these systems has not been accompanied by any educational intervention(s), any systematic evaluation of pre-existing sign-out processes, or by any longterm systematic assessment of the effect of the systems on communication and patient safety. It is possible that although these systems may increase resident efficiency and satisfaction with the sign-out process, they may increase rather than decrease miscommunications. Technological solutions can facilitate well-designed sign-out processes, but they cannot substitute for successful communication. ${ }^{22}$ Effective verbal communication will almost certainly remain crucial to ensure proper transmission of essential clinical information and facilitate collaborative cross-checking. ${ }^{14} 23$

\section{SUMMARY}

The results of our study indicate sign-out between resident physicians is often inadequate and incomplete. The data suggest these deficiencies are not related to the specific role of the resident physician giving or receiving sign-out, how busy those residents are while they are on-call, or how ill the patients the residents are caring for are while they are on-call. Perhaps these deficiencies are due to exchange of the wrong information during the sign-out process. A number of residents commented on the importance of including contingency plans as well as the rationale for the plan of care during sign-out so that if changes need to be made during an on-call shift, there is a clear context for how to best make those changes. This type of information is often not included during sign-out, and increasingly, this kind of information is difficult to find in the medical record. The present study found that nearly a third of the time, residents could not find answers for questions arising while they were on call and they had to rely on their best clinical judgment. Future studies should be directed at identifying the information physicians need while they are on-call and using this information to clearly describe the goals and characteristics of concise and complete sign-out. Studies are also needed to identify how to best teach and evaluate a physician's ability to sign-out.

Acknowledgements: We acknowledge the kind and compassionate young men and women who comprise the paediatric housestaff at the University of Virginia, without whom this work could not have been conceptualised or completed.

Funding: This work was supported in part by funding from the Graduate Medical Education Innovation Program at the University of Virginia and the Association of Pediatric Program Directors Special Project Grant.

Competing interests: None.
2. Charap M. Reducing resident work hours: unproven assumptions and unforeseen outcomes. Ann Intern Med 2004;140:814-5.

3. Perry S. Transitions in care: studying safety in emergency department signovers. Focus on Patient Safety 2004:7:1-3.

4. Leora I, Horwitz, Harlan M, et al. Transfers of patient care between house staff on internal medicine wards: a national survey. Arch Intern Med 2006;166: $1173-7$.

5. Philibert I, Leach DC. Re-framing continuity of care for this century. Qual Saf Heath Care 2005:14:394-6.

6. Lofgren RP, Gottlieb D, Williams RA, et al. Post-call transfer of resident responsibility. J Gen Intern Med 1990;5:501-5.

7. Brown PJ, Borowitz SM, Novicoff W. Information exchange in the NICU: what sources of information do physicians prefer to use. Int $J$ Med Informatics 2004; 73:349-55.

8. Patterson ES, Roth EM, Woods DD, et al. Handoff strategies in settings with high consequences for failure: lessons for health care operations. Int J Qual Health Care 2004;16:125-32.

9. Van Eaton EG, Horvath KD, Lober WB, et al. Organizing the transfer of patient care information: the development of a computerized resident sign-out system. Surgery 2004:136:5-13.

10. Coiera E. When conversation is better than computation. J Am Med Inform Assoc 2000;7:277-86

11. Parker J, Coiera E. Improving clinical communication. J Am Med Inform Assoc 2000; 7:453-61.

12. Lee LH, Levine JA, Schultz HJ. Utility of a standardized sign-out care for new medical interns. J Gen Intern Med 1996:11:753-5.

13. Wilson RM, Runciman WB, Gibberd RW, et al. The quality in Australian health care study. Med J Aust 1995;63:458-71.

14. Arora V, Johnson J, Lovinger D, et al. Communication failures in patient sign-out and suggestions for improvement: a critical incident analysis. Qual Saf Health Care 2005:14:401-7.

15. Sutcliffe KM, Lewton E, Rosenthal MM. Communication failures: an insidious contributor to medical mishaps. Acad Med 2004;79:186-94.

16. Volpp KGM, Grande D. Residents' suggestions for reducing errors in teaching hospitals. N Engl J Med 2003;348:851-5.

17. Patterson ES, Roth EM, Render ML. Handoffs during nursing shift changes in acute care. Proceedings of the Human Factors and Ergonomics Society Meeting 2005:1057-61.

18. Leonard M, Graham S, Banacum D. The human factor: the critical importance of effective teamwork and communication in providing safe care. Qual Saf Health Care 2004;13(Suppl 1):85-90

19. Sharit J, McCane L, Thevenin DM, et al. Examining issues in communicating patient care information across shifts in a critical care setting. Proceedings of the Human Factors Ergonomics Society Meeting 2005:1062-6.

20. Peterson LA, Orav EJ, Teich JM, et al. Using a computerized sign-out program to improve continuity of inpatient care and prevent adverse events. J Oual Improv 1998:24:77-87.

21. Van Eaton EG, Horvath $\mathrm{KD}$, Lober WB, et al. A randomized trial evaluating the impact of a computerized rounding and sign-out system on continuity of care and resident work hours. J Am Coll Surg 2005;200:538-45.

22. Rosenblum ST, Grande J, Geissbuhler A, et al. Experience in implementing inpatient clinical note capture via a provider order entry system. J Am Med Inform Assoc 2004:11:310-6.

23. Patterson ES, Woods DD, Cook Rl, et al. Collaborative cross-checking to enhance resilience. Cogni Tech Work 2007:9:155-62.

\section{Appendix A}

\section{Post-call survey}

Name:

Today's date:

Post-call survey: We are conducting a short survey to better understand our current sign-out process. Please answer the following questions based on your most recent call night.

ALL individual results will remain strictly confidential and when entered into the database, they will be anonymous.

(1) How would you rate your most recent call night? Please circle the correlating number.

\begin{tabular}{lllll}
\hline 1 & 2 & 3 & 4 & 5 \\
Slow & & Medium & & Busy \\
\hline
\end{tabular}

- How many patients were you responsible for caring for when you started call?

$\overline{\text { How }}$ many patients did you admit while you were on call?

How many patients did you transfer to the PICU while you were on call? 
(2) Did anything happen while you were on call that you were not adequately prepared for after sign-out?

No _ Yes

If you answered Yes to question 2, please pick the most important incident that happened during your call night to answer questions 3 through 8 , otherwise skip to question 9 .

(3) Was there information that would have been useful that you did not receive during sign-out?

No If you answered No, please skip to question 7.

Yes - If you answered Yes, please continue with question 4.

(4) Where did you go to get that information that you did not receive during sign-out? (Check all that apply)

The chart:

resident progress note

attending physician note

consultant note

the bedside chart

other

The computer:

MIS*

CareCast†

other

Made it up as it best fit clinically

Asked patient and/or patient's family

I couldn't get it

Other source not listed here, please describe

$\overline{\text { Made }}$ a phone call to:

an attending physician

a fellow

the chief resident

a consultant

somebody else

(5) Which information source was most useful in getting the information you required?

The chart:

resident progress note

attending physician note

consultant note the bedside chart

other

The computer:

MIS*

CareCast†

other

Making it up as it best fit clinically

Asking the patient and/or patient's family

I couldn't get it

Other source not listed here, please describe

A phone call to:

an attending physician

a fellow

the chief resident

a consultant

somebody else

(6) Should this situation have been anticipated and discussed during sign-out?

No Yes

(7) Had you previously cared for this patient (either during a previous call night or a previous day shift)?

No Yes

(8) Did you write a cross cover note (not a daily progress note) about this situation in the chart? No Yes

(9) Overall, how would you rate the sign-out you received at the beginning of your call night?

\begin{tabular}{|c|c|c|c|c|}
\hline 1 & 2 & 3 & 4 & 5 \\
\hline $\begin{array}{l}\text { Inadequate to } \\
\text { answer call } \\
\text { questions }\end{array}$ & & & & $\begin{array}{l}\text { Adequate to } \\
\text { answer call } \\
\text { questions }\end{array}$ \\
\hline
\end{tabular}

(10) Do you have any other comments or suggestions about sign-out that you would like to share at this time?

*MIS, Medical Information System, a hospital-wide computerised physician order entry system.

†CareCast, computerised results and document repository. 